Cómo citar este artículo: Borjas, M. P., Navarro-Lechuga. E., Puentes-Ospino, D., De la cruz-García, J., Yepes-Martínez, J., Muñoz-Alvis, A., Montero, P., De La Hoz-Del Villar, K., Pérez-Moyano, Y., \& Polo, J. D. (2019). Experiencias ludoevaluativas en el contexto universitario: la evaluación desde una comunidad de aprendizaje. Rev.investig.desarro.innov., 10 (1), 177-190. doi 10.19053/20278306.v10.n1.2019.10021

\title{
Experiencias ludoevaluativas en el contexto universitario: la evaluación desde una comunidad de aprendizaje
}

\section{dic-evaluative experiences in the university context: the evaluation from a learning community}

\author{
Mónica Patricia Borjas' \\ Edgar Navarro-Lechuga² \\ Dailin Puentes-Ospino ${ }^{3}$ \\ Johana De la cruz-García ${ }^{4}$ \\ Julián Yepes-Martínez ${ }^{5}$ \\ Andrés Muñoz-Alvis ${ }^{6}$ \\ Pedro Montero 7 \\ Karla De La Hoz-Del Villar8 \\ Yuddy Pérez-Moyano ${ }^{9}$ \\ Jean David Polo ${ }^{10}$ \\ Recibido: noviembre 15 de 2018 \\ Aceptado: junio 14 de 2019
}

\section{Resumen}

La ludoevaluación es una práctica evaluativa que busca valorar los desempeños de los estudiantes a través de experiencias lúdicas, que permitan obtener información oportuna y pertinente sobre sus aprendizajes y reducir los niveles de ansiedad, propios de las experiencias de evaluación tradicional. El objetivo del estudio fue comprender las dinámicas que se generan al realizar experiencias de evaluación, mediadas por la lúdica, en el contexto de una comunidad de aprendizaje docente universitaria. Se trató de una investigación con diseño descriptivo, en la que se desarrollaron experiencias de ludoevaluación con 160 estudiantes de una Universidad privada colombiana. Estas experiencias se llevaron a cabo en 5 fases: fundamentación teórica, diagnóstico,

\begin{abstract}
Ludic Evaluation is an assessment practice that aims to assess students' performance through playful experiences which offer timely and relevant information about their learning and reduce levels of anxiety that are usually found in traditional assessment experiences. The objective of the present study was to understand the classroom interactions that are generated when performing evaluation experiences mediated by games in the context of a university teaching learning community. It was a descriptive study in which experiences of self-assessment were carried out with 160 students at a private Colombian university. These experiences were carried out in 5 phases: theoretical foundation, diagnosis, design, application and reflection. Results show a change
\end{abstract}

1 Licenciada en Educación con énfasis en Biología y Química, Doctora en Diseño Curricular y Evaluación Educativa, Universidad del Norte, Barranquilla, Colombia. E-mail: mborjas@uninorte.edu.co ORCID: 0000-0001-7823-477X.

2-10 Universidad del Norte, Barranquilla, Colombia 
diseño, aplicación y reflexión. Los resultados evidencian un cambio en la percepción de los estudiantes frente a los procesos evaluativos, sobresaliendo emociones positivas, como: el entusiasmo, la alegría y la seguridad. Se concluye que es posible valorar los aprendizajes en escenarios menos estresantes a los que habitualmente se realizan, propios de la evaluación tradicional de corte normativo y cuantitativo.

Palabras clave: ludoevaluación, evaluación formativa, lúdica, comunidad de aprendizaje.

\section{Introducción}

Existe la idea que evaluar es sinónimo de calificación. Sin embargo, llegar a este punto es reducir en gran medida el verdadero valor de la evaluación, cuyo significado va más allá de los procesos de comparación y medición (García, Pueyo \& Gutiérrez, 2013; Quaigrain \& Arhin, 2017). Esta mirada de la evaluación se ampara en un enfoque técnico e instrumental.

Desde el ámbito educativo, la evaluación debe ser ante todo una experiencia humanizante, significativa y con sentido formativo. Esta mirada se orienta por enfoques prácticos y emancipadores. Para Elizondo y Fonseca (2018); García, Martínez, Jaén y Tapia (2016), la evaluación es un proceso que busca recolectar información sobre el aprendizaje y progreso académico de los estudiantes. En ese sentido la evaluación requiere de nutrirse de múltiples fuentes que permitan informar de manera pertinente y oportuna sobre los desempeños, logros y retrocesos de los estudiantes desde todas sus dimensiones, haciendo énfasis en su in the perception of students of the evaluative processes, in which positive emotions such as enthusiasm, joy and safety were highlighted. Based on these results, it was concluded that it is possible to assess learning in less stressful scenarios than those usually found in traditional normative and quantitative assessment.

Keywords: ludic evaluation, formative assessment, ludic, learning community. propósito pedagógico y formativo, pues de ella se derivan las decisiones para el mejoramiento tanto de la enseñanza como de los aprendizajes de los estudiantes.

En el ámbito de la educación superior, desafortunadamente la tendencia alrededor de la evaluación se vincula a modelos tradicionales que favorecen enfoques técnicos, desde donde se privilegia la evaluación cuantitativa y normativa (Koenen, Dochy \& Berghmans, 2015; Gutiérrez, Pérez \& Pérez, 2013; Hortigüela, Pérez \& López, 2015; Córdoba, López \& Sebastiani, 2018; Yepes-Martínez, 2017b). Desde este enfoque el propósito es verificar aprendizajes y desempeños, ubicando a los estudiantes en niveles según los resultados obtenidos a través de diversas técnicas evaluativas, la mayoría de carácter sumativo. Entre estas técnicas se destacan los exámenes, los cuales evidencian resultados en una dimensión limitada del ser humano (principalmente la cognitiva) centrados esencialmente en el saber conocer, dejando de lado la comprensión de otros procesos relacionados con el saber hacer y especialmente el saber ser y convivir. 
A lo anterior se suman estudios como los de Ličanin, Paralija, Čengić, Šurković y Redžic (2015), González, Hernández y Torres (2017), y Duraku, (2017), quienes exponen que si bien los niveles de ansiedad de los estudiantes universitarios tienden a ser elevados debido al reto que presenta esta nueva etapa, estos son aún más elevados durante los exámenes, por la presión de obtener buenas calificaciones. Igualmente, Shibli et al. (2015), Álvarez, Aguilar y Lorenzo (2012), Casari, Anglada y Daher (2014), Carbacho, Torres, Rodríguez y Díaz (2017), y González et al. (2017), coinciden en que durante el período de exámenes, los niveles de ansiedad y estrés en los estudiantes universitarios son elevados, lo cual repercute en los bajos rendimientos académicos. Lo anterior genera la necesidad de crear estrategias de evaluación de corte formativo innovadoras, que permitan además de conocer y retroalimentar los desarrollos de los estudiantes en cuanto a los objetivos o las competencias propuestas, generar un clima de confianza que pueda reducir los efectos negativos de las estrategias tradicionales.

Gaete (2011), Botero (2011), Anadón, Arias, Fernández y González (2017), y Rajeswaran (2019), utilizan estrategias como los juegos de roles que pretenden encontrar un equilibrio entre la lúdica y el aprendizaje. Esos estudios demuestran que el juego tiene aplicabilidad en entornos educativos, haciendo posible desarrollar y evaluar habilidades como la cooperación, la toma de decisiones y la confianza en el otro. Gebremichael (2016), y Alférez, Samos, Ochoa y Quiles (2010), por su parte, apoyan el uso del juego y de las tecnologías como base para el desarrollo de aprendizajes y su evaluación. Afirman que dichas estrategias ayudan a los estudiantes a tener un mejor desempeño en sus exámenes, al tiempo que fortalecen las habilidades para el trabajo en equipo y la cooperación, generando en los estudiantes un mayor compromiso con la clase.

Las experiencias formativas que incluyen el componente lúdico, y revisadas en esta investigación, se asocian especialmente a procesos de enseñanza y en escasa medida a la evaluación. En este sentido, Borjas (2013), ha desarrollado avances alrededor de la interacción evaluación y lúdica en su propuesta de ludoevaluación. La ludoevaluación se considera una experiencia que posibilita conocer los intereses, aprendizajes, desarrollos, así como los obstáculos que limitan el proceso formativo de los estudiantes en contextos lúdicos. Según Borjas (2013), la ludoevaluación promueve la toma de decisiones y la aplicación de los constructos teóricos en contextos retadores, favoreciendo la oportuna retroalimentación realizada a los estudiantes en un ambiente grato, enriquecido por principios como: la lúdica, la cooperación, el diálogo y la motivación.

En la Universidad del Norte, Colombia, se han realizado experiencias de Ludoevaluación para evaluar competencias específicas profesionales en diversas áreas del conocimiento, como: Teoría y Diseño Curricular, Desarrollo del Lenguaje (adelantadas por los docentes Borjas, Flórez \& Astorga, en 2016); Cálculo (adelantada por los docentes Cervantes, Borjas \& Martínez en 2017); o Termodinámica (YepesMartínez, 2017a). Los juegos de roles, los juegos de mesa, los rallys deportivos y las ferias de proyectos, entre otras, han sido mediaciones que posibilitaron la puesta en escena de los principios de la evaluación formativa. Estas estrategias permiten conocer los logros o retrocesos de los estudiantes frente a su proceso de aprendizaje, así como la promoción de procesos de metacognición y de trabajo colaborativo.

En coherencia con lo anterior y con el fin de promover la evaluación formativa en contextos universitarios, en 2015 se conformó en la Universidad del Norte, la comunidad de aprendizaje de ludoevaluación.

Para Bolívar (2014), y Beltrán, Martínez y Torrado (2015), una Comunidad de Aprendizaje Docente (CAD), es un espacio de diálogo entre profesionales reflexivos que se congregan ante un problema común, con la finalidad de trans- 
formar la realidad educativa, comprometiéndose con un aprendizaje a largo plazo. En el caso de la CAD de Ludoevaluación de la Universidad del Norte, el propósito central fue analizar críticamente el valor de la evaluación en el contexto de la formación del futuro profesional, diseñando experiencias de ludoevaluación para aplicarlas en las clases de los docentes integrantes de dicha comunidad.

En este contexto, el objetivo de la presente investigación fue caracterizar las percepciones de los estudiantes con respecto a una experiencia de ludoevaluación en educación superior, adelantada por la CAD de la Universidad del Norte.

\section{Metodología}

El presente trabajo optó por un diseño descriptivo en coherencia con el objetivo de la investigación. Este diseño busca caracterizar y detallar un fenómeno o un grupo, de modo que permita apreciar, de manera amplia y lo más completa posible, la realidad (Ramos, 2015; Eiris \& Gheisari, 2019).

Las experiencias ludoevaluativas se adelantaron en el contexto de la Educación Superior. Participaron un total de siete docentes y 160 estudiantes, de diferentes programas académicos, de la Universidad del Norte, Barranquilla, Colombia. La distribución de la población se presenta en la tabla 1 .

Tabla 1. Participantes en la investigación.

\begin{tabular}{llll}
\hline Programa & Asignatura & Semestre & No. Estudiantes \\
\hline Lic. Pedagogía infantil & Evaluación educativa & VI & 27 \\
Maestría en enseñanza del & Enfoque metodológico de la ense- & Maestría & 13 \\
inglés & ñanza & VI & 24 \\
Derecho & Procedimiento civil especial & VI & 41 \\
Medicina & Epidemiología general & V & 14 \\
Ingeniería Industrial & Mecánica de fluidos & V & 34 \\
Psicología & Modelos de investigación & VIII & 68 \\
Total & Psicología Organizacional* & & 221 \\
\hline
\end{tabular}

*Participaron en la fase de Diagnóstico pero, por motivos de cronograma, no desarrollaron la experiencia ludoevaluativa.

En el desarrollo de la investigación se tuvieron en cuenta las siguientes fases:

i) Fase de fundamentación teórica: la CAD realizó una revisión bibliográfica sobre los fundamentos de la evaluación en el contexto de la educación superior, sus enfoques y tipología, entre otros aspectos. Se hizo énfasis en el carácter formativo de la evaluación. Se analizaron además investigaciones que relacionan la evaluación con emociones y sentimientos.
Lo anterior, con el fin de contar con las bases teóricas que sustentaron el diseño y aplicación de las experiencias de ludoevaluación.

ii) Fase de diagnóstico: los docentes de la CAD aplicaron un cuestionario que posibilitó indagar la percepción que tienen los estudiantes sobre la evaluación del aprendizaje, así como las emociones que asocian a ella. El Cuestionario Diagnóstico (CD), constó de 11 ítems. En ellos se explora la percepción de los estu- 
diantes sobre el propósito de la evaluación, los aspectos o componentes que generalmente evalúan sus docentes en cada una de las asignaturas, así como las emociones que los estudiantes asocian a la evaluación. Igualmente, se indagó sobre las preferencias lúdicas de los estudiantes para, a partir de ellas, planear las actividades de evaluación que se llevarían a cabo.

iii) Fase de diseño: teniendo en cuenta los resultados del CD y de acuerdo a las preferencias lúdicas de los estudiantes, cada docente realizó el diseño de su experiencia de ludoevaluación. Para ello se apoyaron en un formato que incluía elementos como: identificación de la asignatura, propósito de la experiencia evaluativa mediada por la lúdica, competencia o componentes de la competencia a evaluar, descripción de la experiencia, recursos a utilizar, formas de recolección de información sobre los desempeños de los estudiantes y forma en la que se realizaría la retroalimentación a los estudiantes,. iv) Fase de aplicación y de reflexión: las experiencias de ludoevaluación se realizaron en cada una de las asignaturas orientadas por los docentes pertenecientes a la CAD. Luego se aplicó una encuesta o Cuestionario Final (CF), cuya primera parte es similar a la del CD. Además, contiene preguntas abiertas que permiten conocer las percepciones de los estudiantes sobre la experiencia de ludoevaluación realizada. Esta información sirve especialmente para tomar decisiones que permitan mejorar las futuras experiencias tanto de enseñanza como de evaluación. Cabe anotar que sólo el $75 \%$ de los estudiantes diligenciaron de manera completa la encuesta final.

\section{Resultados y discusión}

\subsection{Fundamento teórico y diagnóstico}

Los integrantes de la CAD consolidaron la síntesis de la revisión teórica realizada alrededor de las características de la evaluación formativa, a la cual se suscribe la Ludoevaluación, como se refleja en la figura 1.

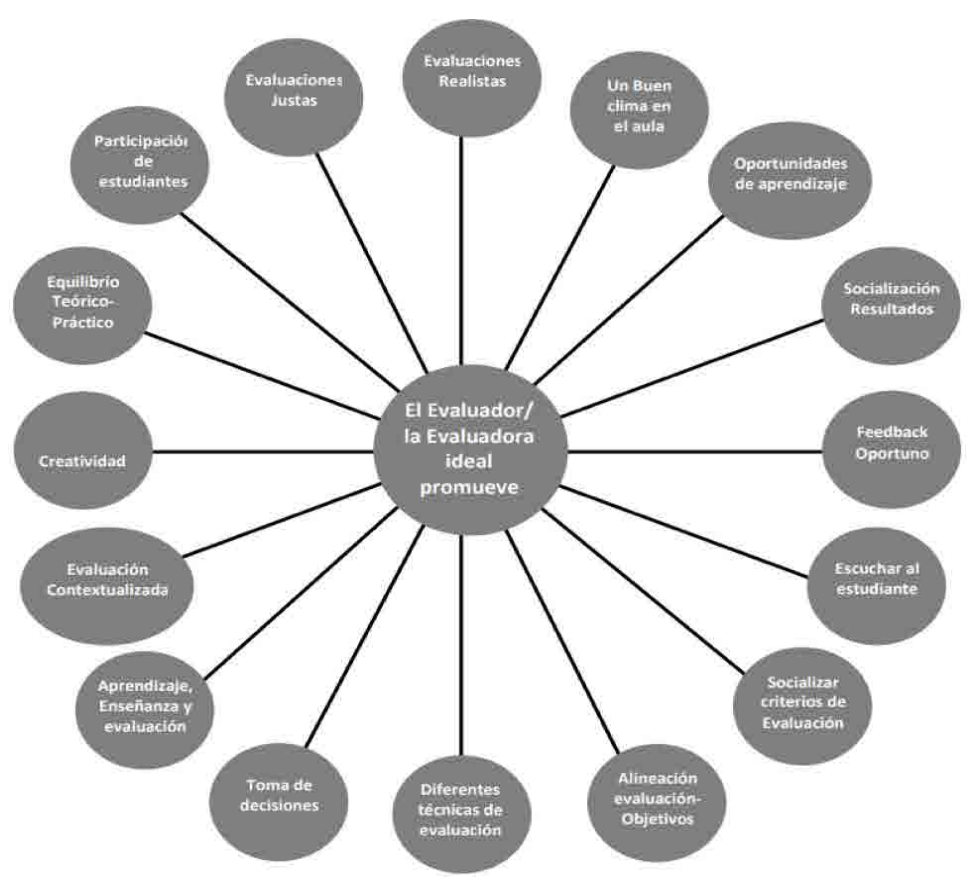

Figura 1. Perfil del evaluador(a) ideal. 
Tal como lo señala Moreno (2015), el perfil profesional del evaluador educativo debe integrar de manera articulada: conocimientos, destrezas, habilidades, valores y actitudes que le permitan ser: proactivo, flexible, oportuno y crítico al: diseñar, desarrollar y evaluar (meta-evaluación) estrategias retadoras, contextualizadas y pertinentes orientadas a: conocer, comprender y valorar cómo aprenden sus estudiantes y a qué responden sus desempeños. Lo anterior con el fin de tomar decisiones que favorezcan tanto los procesos de aprendizaje como los de enseñanza.

El cuestionario inicial de la fase diagnóstica, fue aplicado a un total de 221 estudiantes. De ellos, el $71 \%$, afirma que en su experiencia universitaria, los docentes evalúan con el propósito de verificar sus conocimientos y aprendizajes adquiridos; mientras que un $8 \%$ manifiesta que los evalúan para identificar sus dificultades y fortalezas en el aprendizaje.

Este resultado evidencia la asociación que hacen los estudiantes de la evaluación con el propósito docente de medir los conocimientos. Esto, a su vez, facilita la toma de decisiones para la promoción, basada en los resultados o puntuaciones de los estudiantes, bajo criterios predeterminados y objetivos (Flores-Crespo et al., 2016; Gutiérrez-García, et al., 2013). No obstante, este tipo de evaluación limita la valoración de otros componentes que hacen parte del desarrollo de los seres humanos, y que incluso son mejores predictores del desempeño, en nuestro caso, de los futuros profesionales.
Respecto a las emociones relacionadas con la evaluación, la ansiedad con un $58 \%$, es la más asociada por los estudiantes a este proceso, seguida del miedo (23\%); mientras que emociones como la seguridad (12\%), y el entusiasmo (3\%), son menos asociadas con la evaluación, al igual que la alegría y la tristeza con un $1 \%$, respectivamente.

Las emociones identificadas en el diagnóstico, coinciden con los planteamientos de Feldman et al. (2008), y Álvarez et al. (2012), quienes indican que, en la evaluación tradicional, los niveles de ansiedad durante los exámenes son más altos en comparación con otras actividades. Esto se debe a que los estudiantes perciben la evaluación como un acto en el que sólo se califica y rotula con una nota al estudiante (Martín, 2007). Los trabajos de Martín (2007), Feldman et al. (2008), y Álvarez et al. (2012), muestran que, durante el período de exámenes, los niveles de ansiedad y estrés en los estudiantes universitarios son elevados, lo cual repercute en los bajos rendimientos académicos.

Asimismo, los estudiantes consideran que durante el transcurso de su carrera, en las actividades evaluativas, se ha privilegiado el componente del saber conocer (conceptos, temas, teorías e información), seguido del saber hacer (procedimientos, habilidades y destrezas), y en última instancia el saber ser, el cual hace referencia a actitudes y valores, ver figura 2 . 


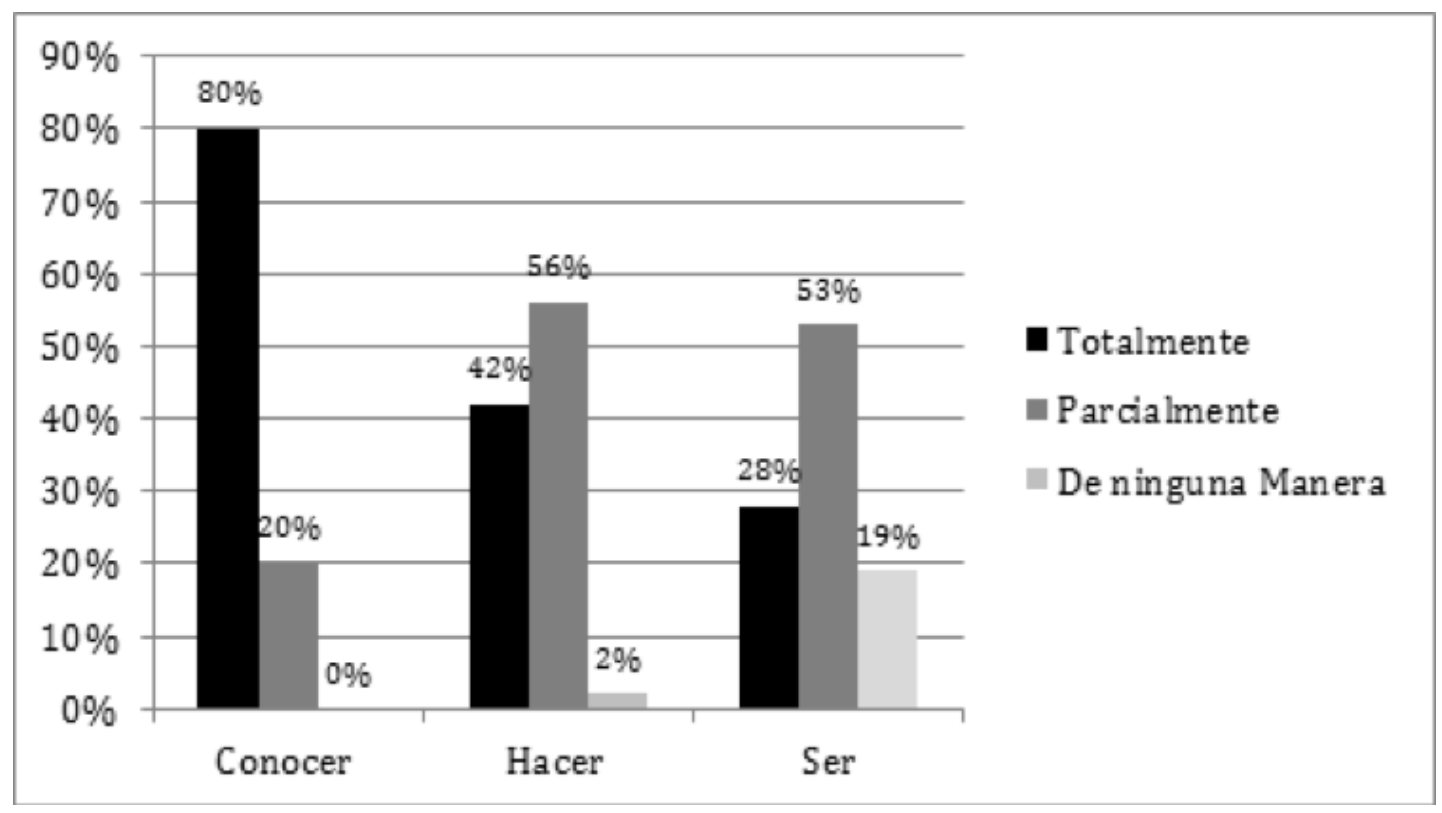

Figura 2. Componentes de las competencias evaluadas según los estudiantes.

\subsection{Diseño}

La revisión teórica y el diagnóstico, permitieron trazar una ruta orientativa para el diseño de las experiencias de Ludoevaluación de los docentes de la CAD. En la tabla 2, se resumen algunas de ellas.

Tabla 2. Experiencias ludoevaluativas adelantadas en las asignaturas.

\begin{tabular}{|c|c|c|}
\hline Asignatura & Nombre de la experiencia & Descripción \\
\hline Evaluación educativa & \multirow{3}{*}{$\begin{array}{l}\text { ¿Quién quiere ser ludo-eva- } \\
\text { luado? (Adaptación del juego } \\
\text { interactivo ¿Quién quiere ser } \\
\text { Millonario?) }\end{array}$} & \multirow[b]{2}{*}{$\begin{array}{l}\text { Se desarrolló un aplicativo para celulares, inspi- } \\
\text { rado en ¿Quién quiere ser millonario?, que busca } \\
\text { evaluar, a través de la ludoevaluación, los compo- } \\
\text { nentes de las competencias específicas de cada } \\
\text { asignatura. }\end{array}$} \\
\hline $\begin{array}{l}\text { Epidemiología general } \\
\text { Mecánica de fluidos } \\
\text { Modelos de investigación }\end{array}$ & & \\
\hline Enfoque metodológico de la & & \\
\hline Procedimiento Civil Especial & Estaciones del Saber & $\begin{array}{l}\text { Los estudiantes, divididos en equipos, elaboran } \\
\text { preguntas sobre los diferentes aspectos de la asig- } \\
\text { natura. Cada equipo se dirige a una estación a fin } \\
\text { de responder las preguntas de los demás equi- } \\
\text { pos. Al responder las preguntas tienen la opción } \\
\text { de seleccionar una pieza de un rompecabezas. El } \\
\text { equipo que arme primero el rompecabezas se lle- } \\
\text { va más puntos. }\end{array}$ \\
\hline
\end{tabular}




\subsection{Aplicación y reflexión}

Luego de realizar las experiencias de ludoevaluación, se procedió a aplicar la encuesta final diligenciada por 160 estudiantes. La mayoría de los estudiantes (46\%), afirma que la experiencia no sólo les permitió verificar o comprobar los conocimientos adquiridos, sino que les posibilitó identificar dificultades y fortalezas en su proceso de aprendizaje (31\%). Igualmente, estas experiencias les permitieron realizar procesos de metacognición sobre sus aprendizajes y/o competencias, como lo reporta el $8 \%$ de los estudiantes encuestados. Asimismo, les permitió reconocerse como sujetos que pueden transformar su realidad haciendo uso de sus competencias (10\%) y encontrarle sentido a su proceso de aprendizaje (5\%).

Gran parte de los estudiantes considera que la evaluación ludoevaluativa posee propósitos formativos más que sumativos, porque les permite autoevaluarse y realizar un proceso de introspección acerca de sus fortalezas y debilidades frente al desarrollo de la asignatura. Esto es coherente con lo planteado por Borjas (2013), al afirmar que, den- tro de los propósitos formativos de la evaluación, se destaca la promoción del autoconocimiento personal de los estudiantes y la retroalimentación como elemento clave, que aporta al desarrollo de procesos metacognitivos. De esta manera, la ludoevaluación se convierte en una oportunidad para reflexionar críticamente sobre sí mismo, permitiendo identificar fortalezas y aspectos por mejorar, lo que en palabras de Goleman (2008), se traduce en avanzar hacia "la conciencia de uno mismo".

Respecto a las emociones positivas que los estudiantes asociaron con la experiencia de ludoevaluación, coinciden en que el entusiasmo (36\%) es la emoción que prevalece en este tipo de evaluación, seguida de la alegría con $15 \%$. En cuanto a las emociones negativas, comparadas con las mencionadas en el diagnóstico, estas disminuyeron significativamente. Ejemplo de ello es la ansiedad, emoción que los estudiantes asociaban con la evaluación en un $58 \%$, y luego de la experiencia ludoevaluativa se redujo a un $36 \%$. Lo mismo ocurrió con el miedo, que fue asociado con la evaluación en un $23 \%$, mientras que después de la Ludoevaluación disminuyó a un 8\%, ver figura 3.

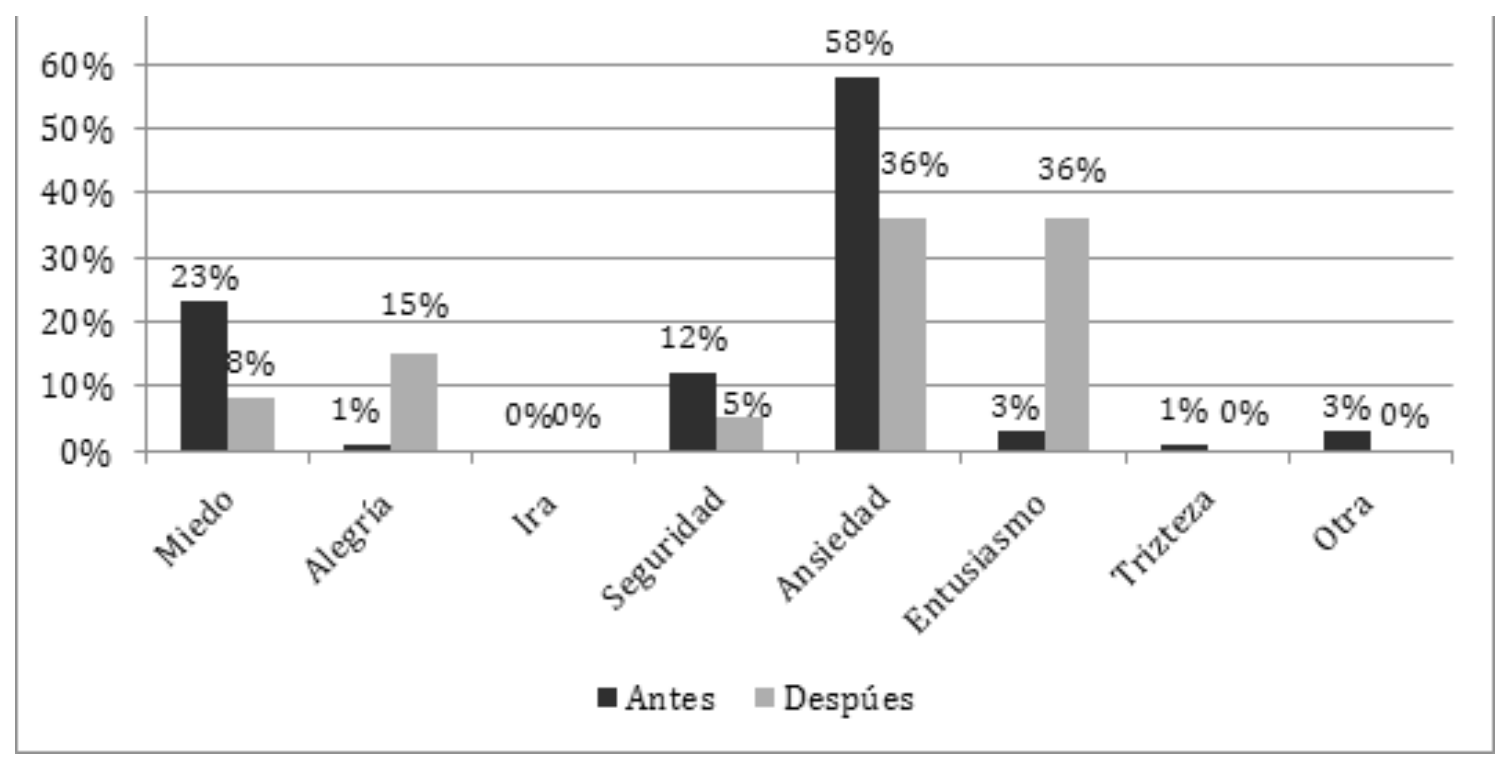

Figura 3. Emociones asociadas a la experiencia ludoevaluativa. 
Los estudiantes consideran que el componente de las competencias relacionado con el saber conocer, es el que está totalmente presente en un evento evaluativo, mientras que los componentes del saber hacer y saber ser, variaron entre los dos momentos en los que fueron encuestados. El cambio más notable fue en relación con el saber ser, el cual incrementó su registro después de la aplicación, ver figura 4.

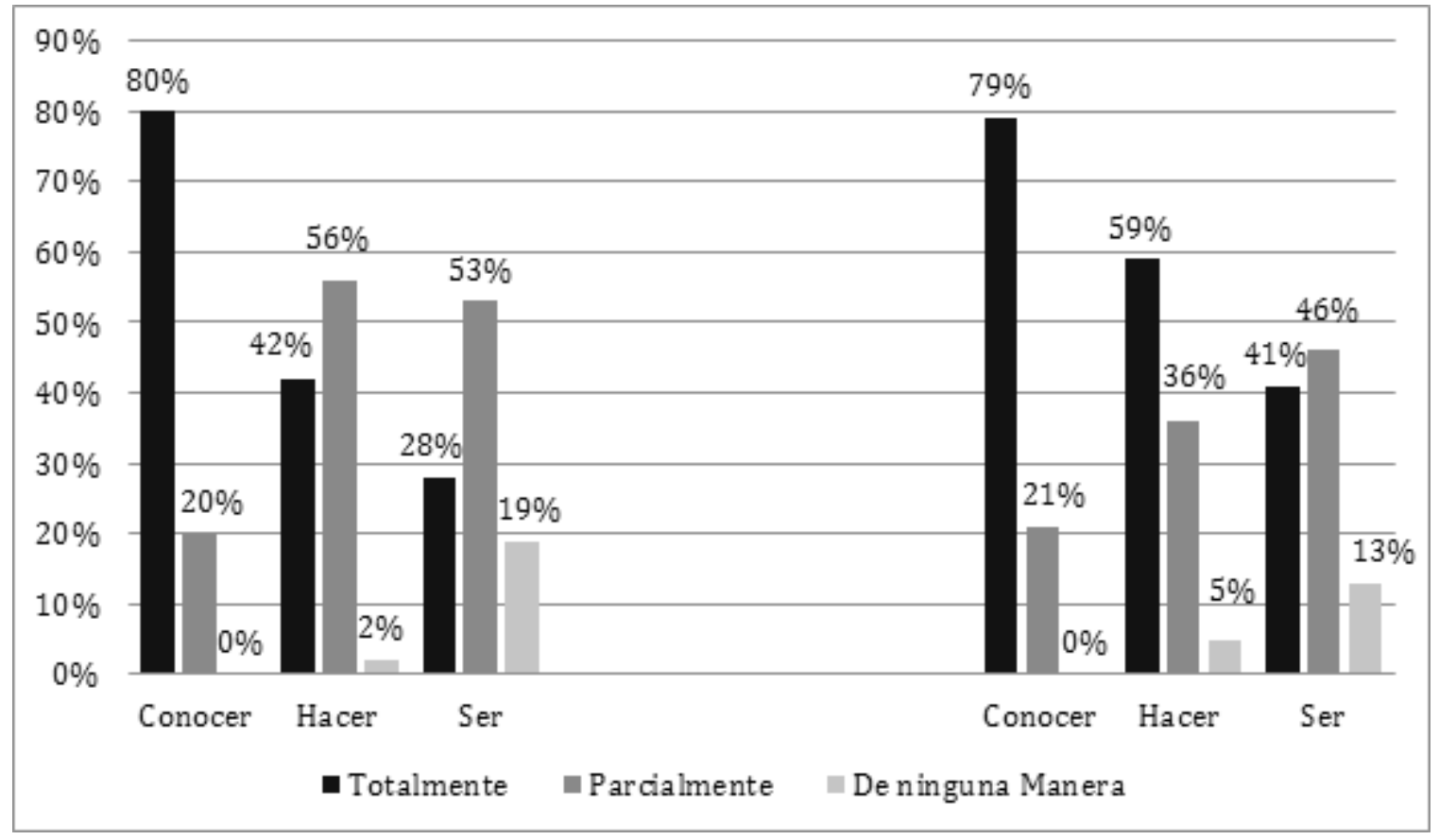

Figura 4. Componentes de las competencias antes y después de la experiencia ludoevaluativa.

Las propuestas tradicionales de evaluación se centran en el conocer, como lo afirma lafrancesco (2005), lo cual también se refleja en los resultados del diagnóstico. Sin embargo, los estudiantes afirmaron que la experiencia ludoevaluativa favoreció un proceso más integral, donde se valoraron los tres componentes de las competencias: saber conocer, saber hacer y saber ser. Se entiende como evaluación integral, el ejercicio que permite evaluar de forma holística todos los procesos relacionados con el aprendizaje de los estudiantes, su desarrollo alrededor de sus dimensiones o la forma como construyen los conocimientos (Chaves, 2015; Yanez, 2016). En este sentido, la evaluación debe considerar: "el aprender a ser, aprender a saber, aprender a saber hacer, aprender a vivir, aprender a convivir, aprender a aprender, aprender a emprender, aprender a sentir, aprender a pensar, aprender a actuar, aprender a liderar" (lafrancesco, 2005).

En cuanto a los principios pedagógicos, la evaluación tradicional privilegia en escasa medida principios como: cooperación, diálogo y lúdica, entre otros. Sin embargo, durante la experiencia de ludoevaluación, estos principios se evidenciaron considerablemente, según lo expresado por más del $80 \%$ de los estudiantes. Los principios más resaltados son: la lúdica y la libertad, con el $100 \%$; seguido de la participación y el autoconocimiento, con el $97 \%$; la cooperación con el $83 \%$ y, finalmente, el diálogo con el $82 \%$.

Al respecto, Borjas (2013), afirma que la ludoevaluación es una experiencia enriquecida por principios como: la cooperación, el diálogo, la par- 
ticipación, la cooperación y la lúdica, siendo este último su eje principal. Para propiciar experiencias basadas en estos principios, Lloyd y Poel (2008), Gaete (2011), Botero (2011), y Anadón et al. (2017), sugieren estrategias como los juegos de roles, con los cuales se pretende encontrar un equilibrio entre el placer por aprender y el aprendizaje. Estos escenarios de evaluación ligados a la lúdica, reducen considerablemente los niveles de ansiedad mejorando el desempeño de los estudiantes (Martín, 2007; Feldman et al., 2008; Álvarez et al., 2012).

Así mismo, se evidencia que al realizar este tipo de evaluación, se genera un aprendizaje significativo haciendo posible aclarar conceptos o procedimientos relacionados con las dinámicas de la clase, como lo manifestaron los estudiantes:

"Aclaré los conceptos básicos que preceden a la ecuación de Bernoulli, y el enfoque práctico en la vida diaria" (Est. Ingeniería Mecánica).

"Sirvió más que para aclarar, que lográramos recordar temas vistos hace ya un tiempo" (Est. Enfoque metodológico de la enseñanza).

Desde la ludoevaluación, como estrategia asociada a la evaluación formativa, es importante conocer cómo esta favorece los procesos de aprendizaje en los estudiantes. En este sentido, la mayoría de los encuestados $(45,62 \%)$ manifestó que la experiencia les permitió autoevaluarse identificando sus fortalezas y limitaciones. A continuación, algunas opiniones de los estudiantes:

"para autoevaluarse en habilidades, conocimientos y actitudes" (Est. Lic. pedagogía infantil).

"Para hacer una autoevaluación de lo que he aprendido" (Est. Medicina).

Igualmente, el $23 \%$ de los estudiantes que participaron de la experiencia ludoevaluativa manifestó que a través de ella pudo reforzar y fortalecer sus conocimientos. En menor medida (1\%), los participantes consideraron que la experiencia permitió poner en escena la toma de decisiones.

En cuanto a recomendaciones para futuras experiencias ludoevaluativas, la mayoría de estudiantes no hicieron sugerencias, por considerar que lo hecho fue adecuado. Sin embargo, algunos de ellos sugieren mejorar los problemas técnicos relacionados con la infraestructura: salones más grandes para desarrollar de manera más libre la experiencia, ajustes a los recursos digitales utilizados o la conectividad, que en ocasiones retrasaron las actividades, así como realizar este tipo de experiencias con mayor frecuencia.

\section{Conclusiones}

Una Comunidad de Aprendizaje Docente (CAD), posibilita el encuentro docente para que, a través del análisis crítico de la realidad educativa, se encuentren opciones orientadas al mejoramiento y la revitalización de las prácticas pedagógicas (Beltrán et al., 2015). Esto es lo que la CAD de ludoevaluación intentó hacer durante un año. La idea de fortalecer las experiencias de evaluación formativa se constituyó en el centro de esta investigación. Los docentes decidieron innovar en sus procesos evaluativos tras identificar en los estudiantes prevención frente a la evaluación.

Luego de la experiencia ludoevaluativa, se evidencia un cambio en la percepción de los estudiantes con respecto a los procesos evaluativos, donde sobresalieron emociones positivas como: el entusiasmo, la alegría y la seguridad. Estos resultados evidencian que es posible valorar los aprendizajes en escenarios menos estresantes a los que habitualmente se realizan, propios de la evaluación tradicional de corte normativo y cuantitativo. Así mismo, se revela la necesidad de crear estrategias innovadoras que permitan reducir los niveles de ansiedad en los estudiantes $y$, a su vez, crear un ambiente propicio para la adquisición y valoración de aprendizajes significativos. 
En consecuencia, se encontró que las experiencias ludoevaluativas permiten a los estudiantes tomar parte activa de su proceso formativo, al tener la oportunidad de mirarse a sí mismos, valorar sus fortalezas y debilidades, autoevaluarse y retroalimentar su propio proceso. Esto es posible gracias a que se privilegia un escenario de: diálogo, cooperación, lúdica y participación activa, que permite ver la evaluación como una oportunidad para reflexionar sobre el aprendizaje, en lugar de ser vista como un mecanismo de verificación y control.

Somos conscientes que una experiencia no puede ser tomada como definitiva para generalizar los resultados. No obstante, con esta investigación pretendemos dar un inicio a futuras intervenciones y motivar a los docentes a atreverse a cambiar las prácticas tradicionales, fundamentándose en procesos de reflexión pedagógica.

\section{Referencias}

Aguilera, A., Mendoza, M., Racionero, S. \& Soler, M. (2010). El papel de la universidad en Comunidades de Aprendizaje. Revista Interuniversitaria de Formación del Profesorado, 24 (1), 45-56. Recuperado de: http://www.redalyc.org/articulo. oa?id=27419180004

Alférez, M. J., Samos, J., Ochoa, J., \& Quiles, J. L. (2010). Desarrollo de un juego como herramienta multimedia de aprendizaje y evaluación. Arspharm, 51 (2), 132-136. Recuperado de: http://digibug. ugr.es/bitstream/handle/10481/27384/Ars\%20 Pharm\%202010\%3b51\%28Suppl2\%29132-136. pdf? sequence $=1$ \&isAllowed $=y$

Álvarez, J., Aguilar, J. M., \& Lorenzo, J. J. (2012). La Ansiedad ante los Exámenes en Estudiantes Universitarios: Relaciones con variables personales y académicas. Electronic Journal of Research in Educational Psychology, 10 (26), 333-354. Recuperado de: http://www.redalyc.org/pdf/2931/293123551017. pdf
Anadón, N., Arias, A., Fernández, I., \& González, M. (2017). Propuesta de utilización del role-playing como medio de evaluación en el Grado en Biología. Revista Infancia, Educación y Aprendizaje, 3 (2), 667-673. doi: https://doi.org/10.22370/ ieya.2017.3.2.799

Beltrán, Y., Martínez, Y., \& Torrado, O. (2015) Creación de una comunidad de aprendizaje: una experiencia de educación inclusiva en Colombia. Revista Encuentros, 13 (2), 57-72. doi: http://dx.doi. org/10.15665/re.v13i2.498

Bolívar, A. (2001). Los centros educativos como organizaciones que aprenden: una mirada crítica. Contexto Educativo-Revista Digital de Educación y Nuevas Tecnologías, 3 (18). 1-11. Recuperado de: https://www.researchgate.net/ profile/Antonio_Bolivar/publication/28068579_ Los_centros_educativos_como_organizaciones_ que_aprenden_Una_mirada_critica/links $/ 55 \mathrm{c} 1 \mathrm{e}$ 0ce08aed9dff2a5794f.pdf

Bolívar, A. (2014). La autoevaluación en la construcción de capacidades de mejora de la escuela como Comunidad de Aprendizaje Profesional. Revista Portuguesa de Investigação Educacional, 14, 9-40. Recuperado de: https://www.researchgate. net/publication/281898047_La_autoevaluacion_ en_la_construccionde_capacidades_de_mejora_de_la_escuela_como_Comunidad_de_Aprendizaje_Profesional

Borjas, M. P. (2013). Ludoevaluación en la educación infantil: más que un requisito, un asunto serio. Barranquilla, Colombia: Editorial Universidad del Norte.

Botero, J. A. (2011). Propuesta de un juego de rol para evaluar la competencia del liderazgo basado en el método de desarrollo de habilidades gerenciales (Tesis doctoral). Universidad Nacional de Colombia, Medellín. 
Carbacho, V. P., Torres, P. A., Rodríguez, M. P., \& DíazNarváez, V. P. (2017). Estado ansioso previo a una evaluación en estudiantes de Odontología. Revista Científica Salud Uninorte, 34 (1), 1-10. doi: http://dx.doi.org/10.14482/sun.34.1.9720

Casari, L., Anglada, J., \& Daher, C. (2014). Estrategias de afrontamiento y ansiedad ante exámenes en estudiantes universitarios. Revista de Psicología, 32 (2), 243-269. Recuperado de: http:// www.scielo.org.pe/scielo.php?script=sci_arttext\&pid=S0254-92472014000200003

Chaves, J. P. (2015). Evaluación, medición o verificación de los aprendizajes en el aula: Un estudio de caso en el Colegio Humanístico Costarricense de Heredia. Revista Electrónica Educare, 19 (2), 405428. doi: https://doi.org/10.15359/ree.19-2.22

Córdoba, T., López, V., \& Sebastiani, E. (2018).Why do I use Formative Evaluation in Physical Education? A Eeacher's Autobiographical Account. Estudios pedagógicos (Valdivia), 44 (2), 21-38. doi: https:// dx.doi.org/10.4067/S0718-07052018000200021

Díez-Palomar, J., \& Flecha, R. (2010). Comunidades de Aprendizaje: un proyecto de transformación social y educativa. Revista Interuniversitaria de Formación del Profesorado, 24 (1), 19-30. Recuperado de: http://www.redalyc.org/articulo. oa?id=27419180002

Duraku, Z. H. (2017). Factors influencing test anxiety among university students. The European Journal of Social \& Behavioural Sciences, 18 (1), 2325. Recuperado de: https://www.futureacademy.org. uk/files/images/upload/ejsbsJanuary2017206.pdf

Eiris, R., \& Gheisari, M. (2019). Site Visit Application in Construction Education: A Descriptive Study of Faculty Members. International Journal of Construction Education and Research, 15 (2), 83-99. doi: 10.1080/15578771.2017.1375050.
Elizondo, J. H., \& Fonseca, A. S. (2018). La evaluación formativa en el proceso enseñanza-aprendizaje en estudiantes de actividad deportiva de la universidad de costa rica. Estudios Pedagógicos, 44 (2), 297310. doi: 10.4067/S0718-07052018000200297

Feldman, L., Goncalves, L., Puignau, C., Zaragoza, J., Bagés, N. \& De Paulo, J. (2008). Relaciones entre estrés académico, apoyo social, salud mental y rendimiento académico en estudiantes universitarios venezolanos. Universitas psychologica, 7 (3), 739-752. Recuperado de: https://revistas.javeriana.edu.co/index.php/revPsycho/article/view/406

Flores-Crespo, P., Blanco, E., Cárdenas, S., Cordero, G., Díaz-Barriga, F., Jiménez, Y., Martínez., \& Ornelas, C. (2016). ¿Por qué no mejora la calidad de la educación básica?. Revista mexicana de investigación educativa, 21 (71), 1295-1303. Recuperado de: http://www.scielo.org.mx/scielo.php?script=sci_arttext\&pid=S1405-66662016000401295\&l$\mathrm{ng}=\mathrm{es} \& \mathrm{t}$ lng $=\mathrm{es}$.

Gaete, R. A. (2011) El juego de roles como estrategia de evaluación de aprendizajes universitarios. Educación y Educadores, 14 (2), 289-307. Recuperado de: http://www.redalyc.org/articulo. oa?id=83421404005

García, Á., Martínez, R., Jaén, J. A., \& Tapia, S. (2016). La autoevaluación como actividad docente en entornos virtuales de aprendizaje/enseñanza. $R e-$ vista de Educación a Distancia, 50, (14). 1-11. doi: http://dx.doi.org/10.6018/red/50/14

Gebremichael, D. (2016). An Evaluation of Gamification to Assess Students' Learning on Their Understanding of First Year Computer Science Programming Module. A dissertation submitted in partial fulfilment of the requirements of Dublin Institute of Technology for the degree of M.Sc. in Computing, Advanced Software Development, 2016 
Goleman. D. (2008). Inteligencia emocional. Barcelona, España: Editorial Kairós

González, L. F., Hernández, A. G., \& Torres, M. T. (2017). Relaciones entre estrés académico, apoyo social, optimismo-pesimismo y autoestima en estudiantes universitarios. Electronic Journal of Research in Education Psychology, 13 (35), 111-130. Recuperado de: https://dialnet.unirioja.es/servlet/ articulo?codigo $=5070708$

Gutiérrez-García, C., Pérez-Pueyo, A., \& Pérez-Gutiérrez, M. (2013). Percepciones de profesores, alumnos y egresados sobre los sistemas de evaluación en estudios universitarios de formación del profesorado de educación física. Ágora para la educación física y el deporte, 2 (15), 130- 151. Recuperado de: http://uvadoc.uva.es/bitstream/ handle/10324/23757/AEFD-2013-2-percepciones-profesores-alumnos.pdf?sequence $=1$ \&isAllowed $=\mathrm{y}$

Hortigüela, D., Pérez, Á., \& López, V. (2015). Implicación y regulación del trabajo del alumnado en los sistemas de evaluación formativa en educación superior. Relieve, 21 (1), ME6. doi: 10.7203/ relieve.21.1.5171

lafrancesco, G. (2005). La evaluación integral y del aprendizaje. Fundamentos y estrategias. Bogotá, Colombia: Escuela Transformadora Magisterio.

Koenen, A. K., Dochy, F., \& Berghmans, I. (2015). A phenomenographic analysis of the implementation of competence-based education in higher education. Teaching and Teacher Education, 50, 1-12. doi: 10.1016/j.tate.2015.04.001

Ličanin, I., Paralija, B., Čengić, D., Šurković, I., \& Redžic, A. (2015). Frequency of anxiety and anxiety disorders among medical students during education. Medical Journal, 21 (4).
Lloyd, P., \& Poel, I. (2008). Designing Games to Teach Ethics. Sci Eng Ethics, 14, 433 - 447. doi: 10.1007/s11948-008-9077-2

Martín, I. (2007). Estrés académico en estudiantes universitarios. Apuntes de psicología, 25 (1), 8799. Recuperado de: https://idus.us.es/xmlui/handle/11441/12812

Moreno-Olivos, T. (2015). Las competencias del evaluador educativo. Revista de la educación superior, 44 (174), 101-126. doi: https://doi.org/10.1016/j. resu.2015.04.011

Quaigrain, K., \& Arhin, A. K. (2017). Using reliability and item analysis to evaluate a teacher-developed test in educational measurement and evaluation. Cogent Education, 4 (1), 2-11. doi: 10.1080/2331186X.2017.1301013

Rajeswaran, C. M. (2019). Task, formulaic language, and role play for developing ESL students' academic language. Indonesian Journal of Applied Linguistics, 8, 536-544. doi: 10.17509/ijal.v8i3.15252

Ramos, C. A. (2015). Los paradigmas de la investigación científica. Avances en Psicología, 23 (1), 9-17. Recuperado de: http://revistas.unife.edu.pe/index. php/avancesenpsicologia/article/view/167/159

Ruiz, E. M. (2005). Creación y desarrollo de comunidades de aprendizaje: hacia la mejora educativa. Revista de educación, 337, 235-250. Recuperado: http://www.revistaeducacion.mec.es/re337/ re337_12.pdf

Shibli, N., Nawaz, N., Ameen, N., Fatima, S., Khan, H. S., \& ul Ain, Q. (2015). The Effects of Anxiety on Achievement and Performance: A College Study. International Journal of Scientific and Research Publications, 5 (6), 2250-3153. Recuperado de: http:// www.ijsrp.org/research-paper-0615/ijsrp-p4292. pdf 
Yanez, P. (2016). El proceso de aprendizaje: fases y elementos fundamentales. Revista San Gregorio, 1 (11), 70-81. Recuperado de: http://oaji.net/articles/2016/3757-1472501941.pdf

Yepes-Martinez, J. (2017a). Ludic evaluation and student engagement in engineering: the case of Thermodynamics. In Conference proceedings of eLearning and Software for Education (eLSE) 2, (01),
234-238. Editura Universităţii Naţionale de Apărare Carol I".

Yepes-Martínez, J. (2017b) Ludoevaluación en la enseñanza de termodinámica en ingeniería. Cándida Filgueira Arias (coord.) Desafíos del paradigma educativo en el siglo XXI: investigación, innovación y formación, 191-194. Madrid, España: Global Knowledge Academics. 